\title{
MRI AND ULTRASOUND CRITERIA FOR THE DIAGNOSIS OF A SPORTS HERNIA IN FOOTBALL PLAYERS
}

DOI: 10.36740/WLek202004125

\author{
Oleksandr Yu. Ioffe ${ }^{1}$, Natalia M. Negriaa ${ }^{2}$, Anastasiia V. Omelchenko ${ }^{1}$, Oleksandr P. Stetsenko ${ }^{1}$, Yuri A. Dibrovaa ${ }^{1}$, \\ Mykola S. Kryvopustov ${ }^{1}$, Yuri P. Tsiura' ${ }^{1}$, Tatiana V. Tarasiuk ${ }^{1}$ \\ '0. O. BOHOMOLETS NATIONAL MEDICAL UNIVERSITY, KYIV, UKRAINE \\ 2CENTER FOR MRI DIAGNOSTICS "M24", KYIV, UKRAINE
}

\begin{abstract}
The aim of the study is to specify diagnostic MRI and ultrasound criteria for a sports hernia in order to verify its diagnosis in football players.

Materials and methods: The study included 50 professional and amateur football players aged 15 to 34 from 2016 to 2019 . The criteria for inclusion in the study were: the presence of groin pain in football players, which prevented them from continuing to actively participate in sports activities.

Results: The findings of the study revealed that during MRI the two factors, which had the strongest influence, were"increased MR signal intensity on PDfs observed from the structures of the inguinal canal" and "increased MR signal intensity on PDfs observed from bone marrow of superior ramus of the pubic bone". During ultrasound of the inguinal area, the main criterion for a sports hernia diagnosis was" increased size of the inguinal canal". The verification of the diagnosis was carried out on the basis of the presence of a protrusion in the posterior wall of the inguinal canal. For a sports hernia diagnosis the MRI sensitivity is $91.67 \%$ ( $95 \% \mathrm{Cl} 77.5-98.2)$, specificity $-78.57 \%$ ( $95 \% \mathrm{Cl} 49.2-95.3)$ and the sensitivity of ultrasound is $88.89 \%$ ( $95 \%$ CI 73.9 - 96.9), the specificity - 50\% (95\% Cl $23.0-77)$.

Conclusions: The combination of MRI and ultrasound makes it possible to accurately detect the presence of a sports hernia in the football player. Based on the findings of our study, we formulated MRI and ultrasound criteria for a sports hernia diagnosis.
\end{abstract}

KEY WORDS: sports hernia, MRI criteria, ultrasound criteria, groin pain syndrome in football players

Wiad Lek. 2020;73(4):755-760

\section{INTRODUCTION}

In most cases, groin pain is the main manifestation of a sports hernia in football players. Physical activity aggravates their pain. In fact, exercise-related painful sensations adversely affect their career [1]. Groin pain is induced by high-intensity explosive rotational movements of the torso and lower extremities, followed by a hard kick [2].

Sports hernia is usually associated with the presence of weakness in the posterior wall of the inguinal canal. The researchers believe that, along with the absence of the anatomical features of a typical hernia, weakness-related prolonged pain in the inguinal area, which aggravates during physical training, can be observed [3].

A general review of the research literature revealed lack of coherent and consistent scientific statements regarding the terminology and diagnostic criteria for reliable assessment of pathological changes in the inguinal area in athletes $[4,5,6]$.

Thus, it is relevant to conduct a research on the specification of diagnostic MRI and ultrasound criteria for a sports hernia.

\section{THE AIM}

The aim of the study is to specify diagnostic MRI and ultrasound criteria for a sports hernia in order to verify its diagnosis in football players.

\section{MATERIALS AND METHODS}

The study included 50 professional and amateur football players aged 15 to 34 with a preliminary diagnosis of a sports hernia. The investigation was conducted at the Department of General Surgery No2 of O. O. Bohomolets National Medical University from 2016 to 2019 . The diagnosis was made on the basis of patient complaints, medical history, clinical test results (according to the guidelines), MRI and ultrasound examination of the groin. The criteria for inclusion in the study were: the presence of pain in the inguinal area of the football players, which prevented them from continuing to actively participate in sports activities, as well as the lack of effect of previous conservative therapy. VAS (the Visual Analogue Scale) was used to assess the pain intensity [7].

The pain impulses were grouped according to the localization of pain, and were defined as follows: the pain experienced within one inguinal area - unilateral (right / left); the pain experienced within both inguinal areas - bilateral. The intensity of pain was determined with the help of the integers between 3 and 10 on the VAS. To verify the diagnosis, all patients with a suspected sports hernia had an ultrasound test and MRI of the inguinal area done. MRI was performed on a $1.5 \mathrm{~T}$ magnetic field induction apparatus using a set of standard pulse sequences. The examination protocol is shown in Table I. 
Table I. MR Inguinal Area Protocol

\begin{tabular}{|c|c|c|c|c|}
\hline Type of weighted image & Proton density (PD) & Proton density (PD) & Proton density (PD) & T1 (T1WI) relaxation time \\
\hline plane & coronal & saggital & axial & coronal \\
\hline Type of sequence & Turbo-spin exo (tse) & Turbo-spin exo (tse) & Turbo-spin exo (tse) & Turbo-spin exo (tse) \\
\hline $\begin{array}{l}\text { Suppression of MRI signal } \\
\text { by adipose tissue }\end{array}$ & + & + & + & - \\
\hline Slice thickness, mm & 3,0 & 3,0 & 3,0 & 3,0 \\
\hline Time of repetition (TR), msec & 2100 & 2100 & 2100 & 610 \\
\hline Time of echo (TE), msec & 33 & 33 & 33 & 11 \\
\hline Field of view (FOV), mm & $180 \times 180$ & $160 \times 120$ & $160 \times 130$ & $160 \times 130$ \\
\hline
\end{tabular}

Table II. Single-factor model coefficients of the risk of a sports hernia diagnosis based on MRI findings on the right

\begin{tabular}{|c|c|c|c|}
\hline Factor & $\begin{array}{l}\text { Coefficient value in the } \\
\text { model, } b \pm m_{b}\end{array}$ & $\begin{array}{l}\text { Level of significance of } \\
\text { difference between the } \\
\text { coefficient and } 0, p\end{array}$ & OR score $(95 \% \mathrm{Cl})$ \\
\hline $\begin{array}{l}\uparrow M R \text { signal from bone marrow of superior ramus } \\
\text { of the right pubic bone }\end{array}$ & $3.3 \pm 1.09$ & $0.002^{* *}$ & $26(3.03-223)$ \\
\hline$\uparrow M R$ signal from the inguinal canal on the right & $3.7 \pm 0.88$ & $<0.001^{* * *}$ & $40.3(7.08-230)$ \\
\hline The size of the inguinal canal on the right & $0.3 \pm 0.11$ & $0.002 * *$ & $1.4(1.13-1.74)$ \\
\hline Lymphatic nodes on the right & $0.16 \pm 0.07$ & $0.03 *$ & $1.17(1.01-1.36)$ \\
\hline $\begin{array}{l}\text { The length of the right rectus abdominis } \\
\text { muscle }\end{array}$ & $0.09 \pm 0.04$ & $0.01 *$ & $1.10(1.02-1.18)$ \\
\hline $\begin{array}{l}\text { The thickness of the right rectus abdominis } \\
\text { muscle }\end{array}$ & $0.08 \pm 0.06$ & 0.189 & $1.08(0.96-1.21)$ \\
\hline$\uparrow M R$ signal from the right adductor muscles & $1.02 \pm 0.68$ & 0.130 & $2.79(0.74-10.6)$ \\
\hline
\end{tabular}

An ultrasound test was performed on a Doppler apparatus with linear and sectoral sensors with a scan frequency of $3.5-5.0 \mathrm{MHz}$. The examination of patients was carried out in a standing and supine position of the patient.

The method of constructing a single-factor and multi-factor logistic regression model was used to identify the link between the risk of a sports hernia in football players and factor variables.

The main criteria for MRI and ultrasound studies of a sports hernia in football players were established based on the analysis of the ROC curve of the multifactor model. The Stepwise method (adding or removing predictor variables) was used to select the minimum set of factors associated with the established diagnosis.

Based on the findings of these studies, the patients were divided into two groups. The first (experimental) group consisted of 36 (72\%) football players who, according to the diagnostic findings, met the established set of diagnostic criteria for a sports hernia. That was a direct indication for surgical treatment in this group. The second group included $14(28 \%)$ football players who were not diagnosed with a sports hernia based on the set of diagnostic criteria for a sports hernia. They were prescribed conservative therapy and kept under continuous monitoring.

\section{RESULTS}

According to MRI data, 21 (63.8\%) patients of the first (experimental) group showed increased MR signal intensity on PDfs observed from the groin structures on both sides, $11(33.3 \%)$ patients - on the right side, $4(22,2 \%)$ patients - on the left side due to the increased hydrophilicity of its structures. Based on the findings of an ultrasound test, all $36(72 \%)$ athletes of the first group had a protrusion in the posterior wall of the inguinal canal (Fig. 1, 2,3).

In the second group, $6(12 \%)$ football players did not have increased MR signal intensity, 8 (16\%) football players had both a slight increase in MR signal intensity along the inguinal canal and increased MR signal intensity at the site of attachment of the adductor muscles, which might be indicative of ARS syndrome. The ultrasound examination did not reveal any changes in the posterior wall of the inguinal canal in all $14(28 \%)$ patients of the second group, but $6(12 \%)$ of them had increased size of the inguinal canal between 11.8 and $12.4 \mathrm{~mm}$. They were prescribed conservative therapy and kept under continuous monitoring.

A single-factor logistic regression model was firstly constructed in order to study the relationship between the risk of a sports hernia in football players and findings of MRI and ultrasound diagnostics.

The results of the coefficient estimation and the calculation of odds ratios (OR) for the logistic regression model, including the right and left side of the body, have been summarized in Tables II, III.

It was found that one of the criteria for the presence of a sports hernia $(\mathrm{p}<0.001)$ was increased MR signal inten- 
Table III. Single-factor model coefficients of the risk of a sports hernia diagnosis based on MRI findings on the left

\begin{tabular}{|c|c|c|c|}
\hline Factor & $\begin{array}{l}\text { Coefficient value in the } \\
\text { model, } b \pm_{m} b\end{array}$ & $\begin{array}{l}\text { Level of significance of } \\
\text { difference between the } \\
\text { coefficient and } 0, p\end{array}$ & OR score $(95 \% \mathrm{Cl})$ \\
\hline $\begin{array}{l}\uparrow M R \text { signal from bone marrow of superior } \\
\text { ramus of the left pubic bone }\end{array}$ & $-0.11 \pm 0.63$ & 0.86 & $0.89(0.26-3.08)$ \\
\hline$\uparrow M R$ signal from the inguinal canal on the left & $0.69 \pm 0.64$ & 0.28 & $2(0.57-7.02)$ \\
\hline The size of the inguinal canal on the left & $0.068 \pm 0.08$ & 0.38 & $1.07(0.92-1.25)$ \\
\hline Lymphatic nodes on the left & $0.18 \pm 0.08$ & $0.02^{*}$ & $1.2(1.02-1.41)$ \\
\hline The length of the left rectus abdominis muscle & $0.09 \pm 0.03$ & $0.01^{*}$ & $1.09(1.02-1.17)$ \\
\hline $\begin{array}{l}\text { The thickness of the left rectus abdominis } \\
\text { muscle }\end{array}$ & $0.04 \pm 0.05$ & 0.43 & $1.04(0.97-1.16)$ \\
\hline$\uparrow M R$ signal from the left adductor muscles & $-0.11 \pm 0.63$ & 0.86 & $0.89(0.26-3.08)$ \\
\hline
\end{tabular}
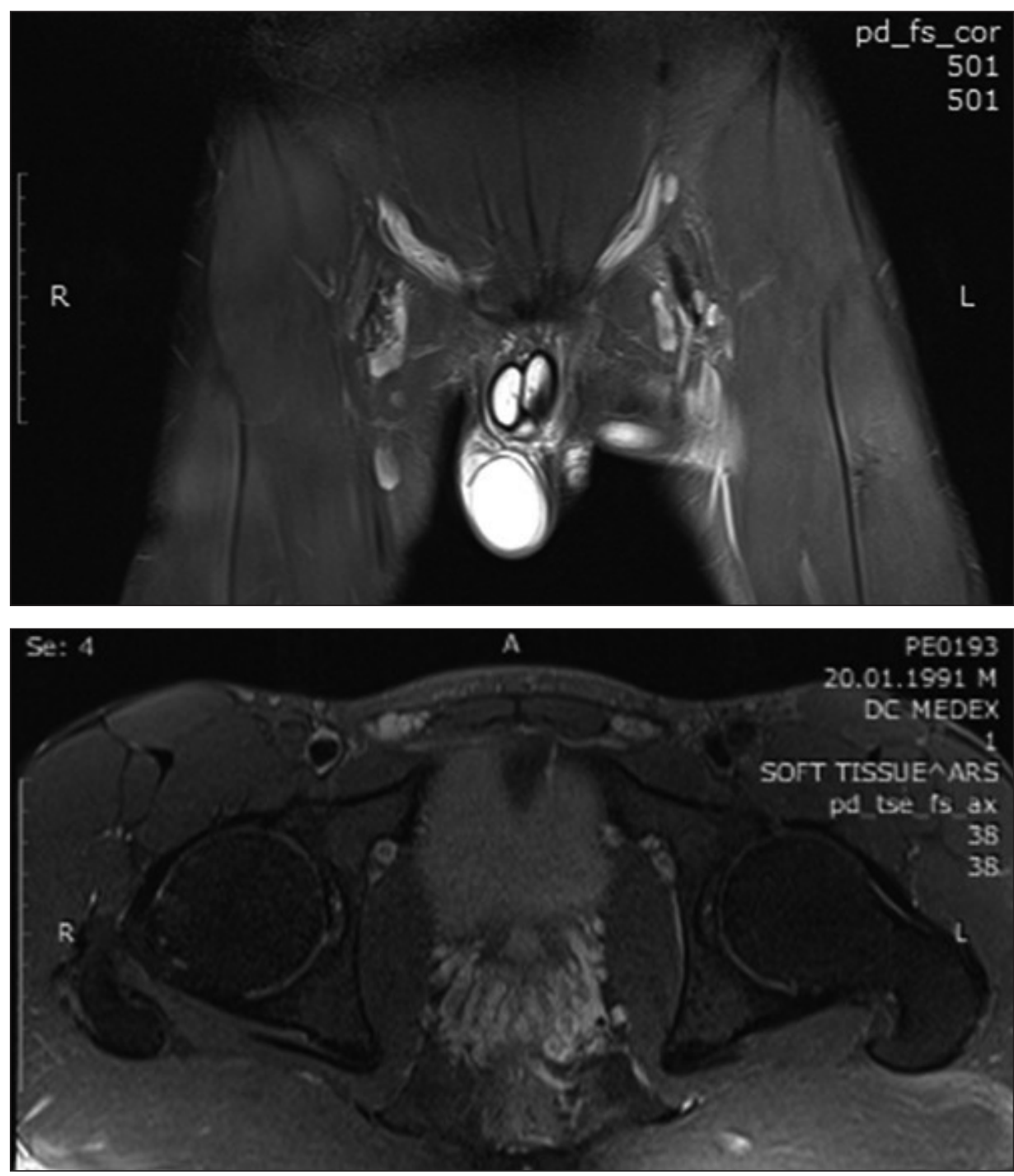

Fig. 1. Increased MR signal intensity observed from the structures of both inguinal canals
Fig. 2. Increased MR signal intensity observed from the structures of both inguinal canals sity on PDfs, which was produced by the structures of the inguinal canal, along with increased MR signal intensity related to the bone marrow of superior ramus of the right pubic bone $(\mathrm{p}=0.003)$. Thus, when increased MR signal intensity is observed from the structures of the inguinal canal on the right, OR 40.3 (95\% CI 7.08 - 230), and from bone marrow of superior ramus of the right pubic bone,
OR 26 (95\% CI 3.03 - 223), the likelihood of diagnosing a sports hernia increases.

It is more likely to diagnose a sports hernia in football players with increased size of the inguinal canal, OR 1.55 (95\% CI 1.01-2.38) on the right and 1.07 (95\% CI 0.83-1.36) on the left. The results of the coefficient estimation and the calculation of odds ratios (OR) for the logistic regression 


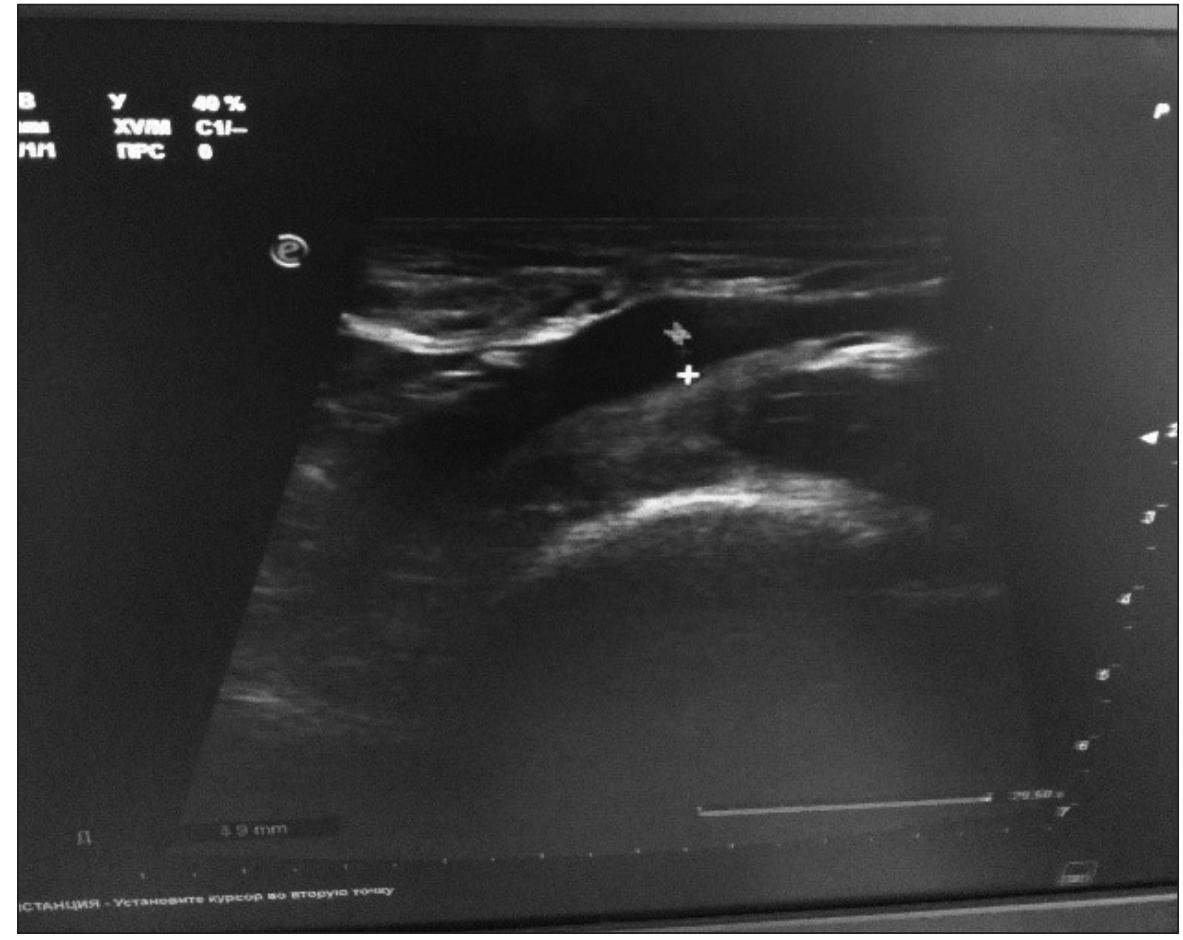

Fig. 3. Sports hernia ultrasound examination. Widening of the inguinal canal and a protrusion of the "weak zone" of the posterior wall of the inguinal canal

Table IV. Single-factor model coefficients for predicting the risk of a sports hernia diagnosis in football players based on ultrasound findings on the right

\begin{tabular}{|c|c|c|c|}
\hline Factor & $\begin{array}{l}\text { Coefficient value in the } \\
\text { model, } b \pm m_{b}\end{array}$ & $\begin{array}{l}\text { Level of significance of difference } \\
\text { between the coefficient and } 0, p\end{array}$ & OR score $(95 \% \mathrm{Cl})$ \\
\hline Inguinal canal on the right & $0.44 \pm 0.22$ & $0.04 *$ & $1.55(1.01-2.38)$ \\
\hline $\begin{array}{l}\text { The size of the deep inguinal } \\
\text { ring on the right }\end{array}$ & $0.06 \pm 0.12$ & 0.71 & $1.07(0.83-1.36)$ \\
\hline
\end{tabular}

model, including the right and left side of the body, have been summarized in Tables IV, V.

It was also noted that, when comparing the factors for a sports hernia diagnosis, a difference was found between the values on the right and left side of the body, which may be explained by individual biomechanical characteristics of athletes and that football players in the first and second groups predominantly use their right lower extremity during the game.

A multifactor logistic regression model was constructed to select MRI and ultrasound factors in order to verify the diagnosis of a sports hernia in football players in the first and second groups. The results of the coefficient estimation in the model are shown in Table VI, VII. Two main criteria were identified during MRI: "increased MR signal intensity on PDfs observed from the structures of the inguinal canal" and "increased MR signal intensity on PDfs observed from bone marrow of superior ramus of the pubic bone", which showed signs of increasing hydrophilicity of the inguinal area structures.

With regard to the inguinal area ultrasound, the main criterion of a sports hernia was "increased size of the inguinal canal". However, the cardinal sign, indicating the presence of a sports hernia, was a protrusion of the posterior wall of the inguinal canal, which was detected in all football players in the first group.
Thus, based on the two main factors that were determined during MRI examination, namely "increased MR signal intensity on PDfs observed from the structures of the inguinal canal" and "increased MR signal intensity on PDfs observed from bone marrow of superior ramus of the pubic bone", a two-factor model was constructed to discover the effect of these factors on the detection of a sports hernia. The model is adequate, the area under the ROC - curve is $\mathrm{AUC}=0.92$ (95\% CI $0.82-0.98)$, statistically significant $(p<0,03)$ and differs from 0,05 . Ultrasound revealed that a growth of one factor increases the risk of a sports hernia detection, namely "increased size of the inguinal gap", the area under the ROC - curve AUC = 0.64 (95\% CI 0.49 - 0.77) (Figure 4, 5).

It was found that the MRI sensitivity is $91.67 \%$ (95\% CI 77.5 - 98.2), specificity -78.57\% (95\% CI 49.2 - 95.3), positive predictive value $\mathrm{PPV}=91.7 \%$, negative predictive value $\mathrm{NPV}=78.6 \%$.

Whereas, for the diagnosis of a sports hernia the sensitivity of ultrasound is $88.89 \%$ (95\% CI $73.9-96.9$ ), the specificity is $50 \%$ (95\% CI $23.0-77)$, positive predictive value $\mathrm{PPV}=82.1 \%$, negative predictive value $\mathrm{NPV}=63.6 \%$.

\section{DISCUSSION}

One of the main imaging methods for the diagnosis of a sports hernia is MRI, which helps to determine structural changes 
Table V. Single-factor model coefficients for predicting the risk of a sports hernia diagnosis in football players based on ultrasound findings on the left

\begin{tabular}{cccc}
\hline Factor & $\begin{array}{c}\text { Coefficient value in the } \\
\text { model, } \mathbf{b} \pm \mathbf{m}_{\mathbf{b}}\end{array}$ & $\begin{array}{c}\text { Level of significance of difference } \\
\text { between the coefficient and } \mathbf{0 ,} \mathbf{p}\end{array}$ & OR score (95\%Cl) \\
\hline Inguinal canal on the left & $0.36 \pm 0.2$ & 0.08 & $1.43(0.95-2.15)$ \\
\hline $\begin{array}{c}\text { The size of the deep inguinal } \\
\text { ring on the left }\end{array}$ & $0.15 \pm 0.17$ & 0.35 & $1.17(0.84-1.62)$ \\
\hline
\end{tabular}

Table VI. Two-factor model coefficients for predicting the risk of a sports hernia diagnosis in football players based on MRI findings

\begin{tabular}{|c|c|c|c|}
\hline Factor & $\begin{array}{l}\text { Coefficient value in the } \\
\text { model, } b \pm m_{b}\end{array}$ & $\begin{array}{l}\text { Level of significance of difference } \\
\text { between the coefficient and } 0, p\end{array}$ & OR score $(95 \% \mathrm{Cl})$ \\
\hline $\begin{array}{l}\uparrow M R \text { signal from bone } \\
\text { marrow of superior ramus of } \\
\text { the right pubic bone }\end{array}$ & $2.68 \pm 1.24$ & 0.03 & $14.6(1.28-166.9)$ \\
\hline $\begin{array}{l}\uparrow M R \text { signal from the inguinal } \\
\text { canal on the right }\end{array}$ & $3.24 \pm 0.98$ & $<0.001 * * *$ & $25.6417(3.74-175.7)$ \\
\hline
\end{tabular}

Table VII. Coefficient of the risk of a sports hernia diagnosis in football players based on ultrasound findings

\begin{tabular}{cccc}
\hline Factor & $\begin{array}{c}\text { Coefficient value in the } \\
\text { model, } \mathbf{b} \pm \mathbf{m}_{\mathbf{b}}\end{array}$ & $\begin{array}{c}\text { Level of significance of difference } \\
\text { between the coefficient and } \mathbf{0 ,} \mathbf{p}\end{array}$ & OR score (95\%Cl) \\
\hline Inguinal canal on the right & $0.44 \pm 0.22$ & $0.04^{* *}$ & $1.55(1.01-2.38)$ \\
\hline
\end{tabular}

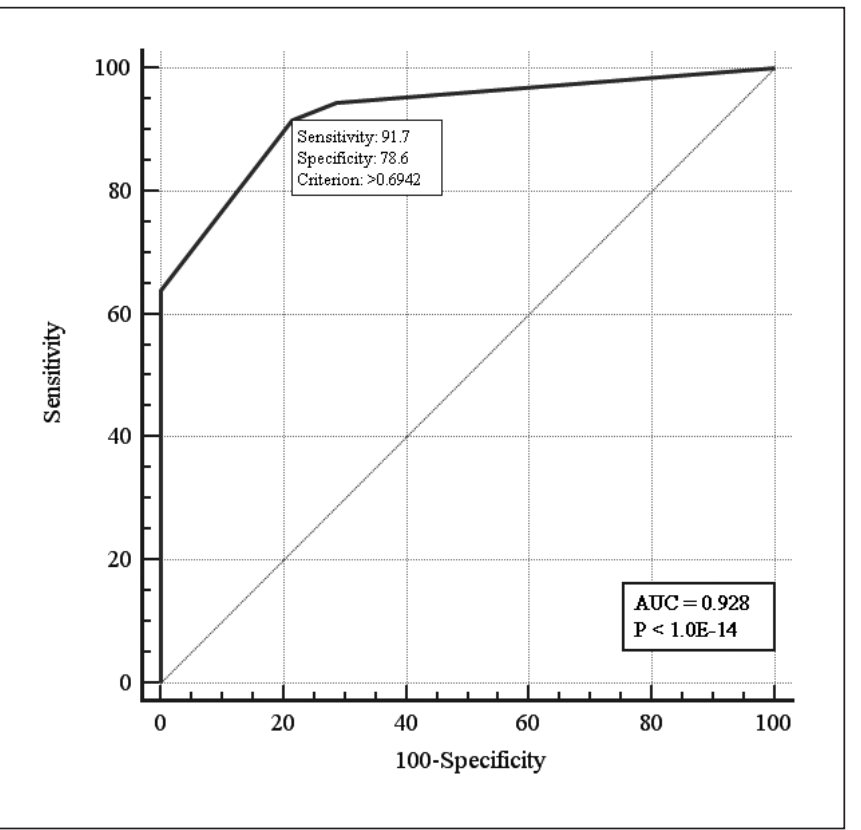

Fig. 4. ROC - curve of the two-factor model for predicting the risk of a sports hernia diagnosis based on MRI findings

in the inguinal canal [8]. In addition, this method provides information on the nature of the changes in the pubic bone and adjacent myotendinosis structures $[9,10,11,12]$. Along with MRI, ultrasound remains a sufficiently informative method for the examination of this part of the human body [13].

MRI and ultrasound findings make it possible to specify anatomical changes in the inguinal area, which may be considered the relevant criteria for diagnosing a sports hernia in football players.

It was also noted that, when comparing the factors for a sports hernia diagnosis, a difference was found between

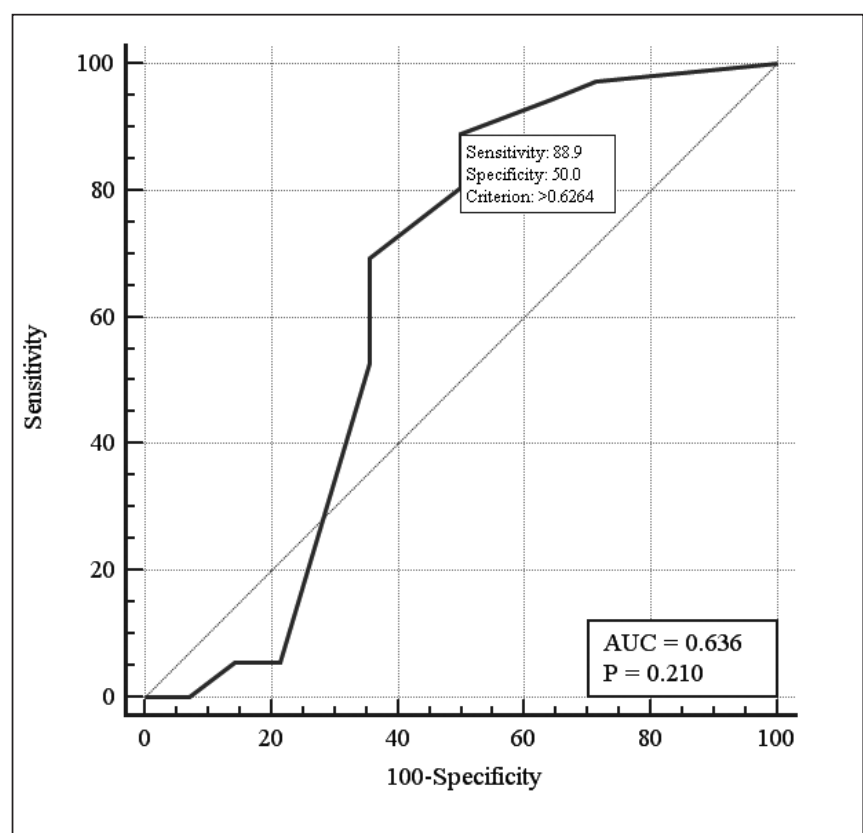

Fig. 5. ROC - curve for predicting the risk of a sports hernia diagnosis based on ultrasound findings

the values on the right and left side of the body, which may be explained by individual biomechanical characteristics of athletes and that football players in the first and second groups predominantly use their right lower extremity during the game.

The findings of the study revealed that during MRI the two factors, which had the strongest influence, were "increased MR signal intensity on PDfs observed from the structures of the inguinal canal" and "increased MR signal intensity on PDfs observed from bone marrow of superior ramus of the pubic bone". During ultrasound of 
the inguinal area, the main criterion for a sports hernia diagnosis was "increased size of the inguinal canal". The verification of the diagnosis was carried out on the basis of the presence of a protrusion in the posterior wall of the inguinal canal, as it was detected in all $36(100 \%)$ patients in the first group.

Most likely, these data indicate pathological changes involving anatomical structures in the inguinal area. These abnormalities develop due to the appearance of signs of reactive changes in the pubic bones, resulting from their displacement one against another in the symphysis area, the spread of oedema to the surrounding soft tissues of the inguinal area with increasing hydrophilicity of the inguinal structures, increased MR signal intensity and signs of inguinal lymphadenopathy.

Based on the findings of our study, we formulated MRI and ultrasound criteria for a sports hernia diagnosis.

\section{CONCLUSIONS}

Increased MR signal intensity on PDfs observed from the structures of the inguinal canal and increased MR signal intensity on PDfs observed from bone marrow oedema of superior ramus of the pubic bone, as well as increased size of the inguinal canal and the presence of the "weak zone" protrusion in the posterior wall of the inguinal canal on ultrasound may indicate the presence of a sports hernia in football players.

MRI and ultrasound are highly informative imaging methods for a sports hernia diagnosis. The combination of these methods makes it possible to accurately detect the presence of a sports hernia in the football player.

\section{REFERENCES}

1. Rennie W., Lloyd D. Sportsmans Groin: The Inguinal Ligament and the Lloyd Technique. J Belg Soc Radiol. 2017; 101 (Suppl 2):16.

2. Chopra A., Robinson P. Imaging Athletic Groin Pain. Radiologic Clinics of North America. 2016; 54 (5): 865-873.

3. Weir A., Brukner P., Delahunt E., et al. Doha agreement meeting on terminology and definitions in groin pain in athletes. British Journal of Sports Medicine. 2015; 49 (12): 768-774.

4. Branci S., Thorborg K., Nielsen M., Hölmich P. Radiological findings in symphyseal and adductor-related groin pain in athletes: a critical review of the literature. British Journal of Sports Medicine. 2013; 47 (10): 611-619.

5. Paajanen H., Hermunen H., Ristolainen L., Branci S. Long-standing groin pain in contact sports: a prospective case-control and MRI study. BMJ Open Sport \& Exercise Medicine. 2019; 5(1): e000507.

6. Khan W., Zoga A., Meyers W. Magnetic Resonance Imaging of Athletic Pubalgia and the Sports Hernia. Magnetic Resonance Imaging Clinics of North America. 2013; 21(1): 97-110.

7. EuraHS Q0L. EuraHS-Quality-of-Life-Score [Internet] 2016 Jun 14. Available from: http://www.eurahs.eu/EuraHS-Quality-of-Life-Score.php

8. Helms C.A., Major N.M., Anderson M.W. et al. Basic principles of musculoskeletal MRI. Musculoskeletal MRI. 2009 (2): 1-19.
9. Branci S., Thorborg K., Bech B., et al. The Copenhagen Standardised MRI protocol to assess the pubic symphysis and adductor regions of athletes: outline and intratester and intertester reliability. British Journal of Sports Medicine. 2014; 49(10): 692-699.

10. Thorborg K., Reiman M., Weir A., et al. Clinical Examination, Diagnostic Imaging, and Testing of Athletes With Groin Pain: An Evidence-Based Approach to Effective Management. Journal of Orthopaedic \& Sports Physical Therapy. 2018; 48(4): 239-249.

11. Lee S., Endo Y., Potter H. Imaging of Groin Pain: Magnetic Resonance and Ultrasound Imaging Features. Sports Health: A Multidisciplinary Approach. 2017; 9(5): 428-435.

12. Campanelli G. Pubic inguinal pain syndrome: the so-called sports hernia. Hernia. 2010; 14(1): 1-4.

13. Kwee R., Kwee T. Ultrasonography in diagnosing clinically occult groin hernia: systematic review and meta-analysis. European Radiology. 2018; 28(11): 4550-4560.

The work is a fragment of the planned research work of the Department of General Surgery No2 of the National Medical University named after O. O. Bohomolets "Implementation of minimally invasive surgical techniques in the treatment of pathologies of the abdominal cavity, anterior abdominal wall, morbid obesity by fast track technique" (State registration number 0118U000147).

\section{Authors contributions:}

Oleksandr Yu. Ioffe - 0000-0002-1306-7920 A

Natalia M. Negria - 0000-0001-8643-6371 ${ }^{B}$

Anastasiia V. Omelchenko - 0000-0001-9968-3750 B,D

Oleksandr P. Stetsenko - 0000-0002-2219-653X ${ }^{E}$

Yuri A. Dibrova - 0000-0002-2833-1667 ${ }^{E}$

Mykola S. Kryvopustov - 0000-0002-8221-5106 ${ }^{C}$

Yuri P. Tsiura - 0000-0001-6651-8564 ${ }^{B}$

Tatiana V. Tarasiuk - 0000-0001-6629-3908 ${ }^{E}$

\section{Conflict of interest:}

The Authors declare no conflict of interest.

\section{CORRESPONDING AUTHOR}

\section{Anastasiia V. Omelchenko}

Department of General Surgery 2

0.0. Bohomolets National Medical University

Taras Shevchenko Boulevard, 13, 01601, Kyiv, Ukraine

tel: +380668374947

e-mail:dr.anastasiia_omelchenko@ukr.net

Received: 03.07.2019

Accepted: 10.02 .2020

A - Work concept and design, B - Data collection and analysis, C - Responsibility for statistical analysis,

D -Writing the article, $\mathbf{E}$-Critical review, $\mathbf{F}$ - Final approval of the article 